\title{
Membrane-based therapeutic plasma exchange in tertiary care ICU: demographic characteristics and predictors of complications
}

\author{
Mitul P Chavda, Alpesh Patel and Shailesh Bihari
}

Membrane-based therapeutic plasma exchange (mTPE) has been used to treat various diseases in the intensive care unit (ICU); $;^{1-11}$ however, there are relatively sparse data available for the feasibility, common indications, and complications of MTPE in the intensive care setting. ${ }^{1-6}$

The most recent American Society for Apheresis (ASFA) guideline for the use of therapeutic apheresis in clinical practice provides guidance on the use of therapeutic plasma exchange (TPE) on a variety of diseases. ${ }^{12}$ However, a limited number of these indications required intensive care support, including Guillain-Barré syndrome, acute liver failure, myasthenic crisis, thrombotic thrombocytopenic purpura, and Goodpasture syndrome.

Generally, mTPE is a safe and well tolerated procedure, and most complications are either mild and do not require intervention or moderate and require treatment, but MTPE can be completed. Severe complications where MTPE needs to be interrupted are rare..$^{13-15}$ The incidence of adverse events during mTPE has been reported as $11 \%$ of all mTPE procedures according to the World Apheresis Association Registry. ${ }^{13}$ However, in the intensive care setting, data regarding complications during MTPE are scarce and reported much higher, up to $45.5 \%$ of mTPE procedures. ${ }^{4}$

There is a lack of Australian data regarding different indications and complications associated with the use of MTPE in the ICU. Therefore, we report our single-centre clinical experience using mTPE at our tertiary care ICU. The objective of our study was to review our MTPE practice and examine factors affecting patient complications and circuitrelated complications in patients undergoing MTPE.

\section{Methods}

The Flinders Medical Centre ICU conducts all mTPE treatment for patients who require plasma exchange in the hospital since April 2014. We prospectively collected data for all ICU as well as non-ICU (ward) patients who required mTPE procedures performed at the Flinders Medical Centre ICU between April 2014 and December 2020. Individual patient consent was exempted by the Southern Adelaide

\begin{abstract}
Introduction: Membrane-based therapeutic plasma exchange (mTPE) has been used to treat various diseases in the intensive care unit (ICU) setting. However, there is a lack of clinical data regarding the practice of MTPE from Australian ICUs.
\end{abstract}

Objectives: To determine factors contributing to complications in patients undergoing MTPE in the ICU.

Methods: Prospectively collected data for mTPE procedures performed at the ICU of Flinders Medical Centre between April 2014 and December 2020 were analysed.

Results: During the study period, 674 mTPE treatments were performed in 140 patients (71 females, 50.7\%). Haematological disease (30.4\%) was the most common indication for mTPE treatment. Citrate was the most common anticoagulation for mTPE $(86.1 \%)$, while albumin $(42.3 \%)$ was the most common replacement fluid. Circuit complications occurred in $18.6 \%$ of the total mTPE treatments. On logistical regression analysis, treatment ionised calcium level (odds ratio [OR], 42.2; $95 \% \mathrm{Cl}, 1.8-975.0 ; P=0.02)$, male sex $(\mathrm{OR}, 2.04 ; 95 \% \mathrm{Cl}$, $1.04-4 ; P=0.04)$, duration of mTPE treatment (OR, 1.02; $95 \% \mathrm{Cl}, 1.01-1.02 ; P<0.001)$ and diagnostic categories $(P=0.03)$ were predictors of circuit complications. During mTPE treatment, $87.2 \%$ of patients did not experience any complications. On logistical regression analysis, replacement fluid type ( $P=0.03$ ), lower initial blood flow (OR, 0.9; $95 \%$ $\mathrm{Cl}, 0.9-1.0 ; P=0.04)$ and higher exchange volume (OR, $8.9 ; 95 \% \mathrm{Cl}, 1.6-48.7 ; P=0.01)$ were predictors of patient complications.

Conclusion: During mTPE, pre-treatment ionised calcium level, male sex, duration of mTPE and diagnostic categories were predictors of circuit complications, while replacement fluid type, initial blood flow and higher exchange volume were predictors of patient complications.

Crit Care Resusc 2022; 24 (1): 43-9 


\section{ORIGINAL ARTICLES}

Clinical Human Research Ethics Committee, as our study was approved as a quality service improvement project.

All MTPE procedures were performed using the Prismaflex (Baxter) continuous renal replacement therapy machine using the TPE Prismaflex 2000 plasma exchange filter (adult), which has a polypropylene membrane with $0.35 \mathrm{~m}^{2}$ filter surface area. The MTPE procedure was performed by ICU nurses and treatment was prescribed by the ICU consultant. Choice of anticoagulation, exchange volume, and replacement fluid was decided by the patient's indication and clinical judgement.

\section{Data source}

A total of 140 patients with 674 mTPE treatments were identified from the mTPE data sheet filled by ICU consultants and ICU nurses (Online Appendix, figure 1). The data sheet for MTPE include the patient's demographic characteristics; mTPE treatment information, such as indication, timing, treatment order, procedure details and circuit; and patient complications.

The patients' demographic data collected included age, sex, weight, indications of MTPE, and numbers of mTPE. The mTPE procedure-related data reported included duration, replacement fluid type, exchange volume, mode of anticoagulation, blood flow, ionised calcium level before and after treatment, and filtration fraction.

From the MTPE data sheet, we identified and recorded complaints reported by patients, clinical symptoms, deterioration in vital signs, mTPE-related problems, and change in laboratory value of ionised calcium. Circuit complication was defined as any reason that required change of mTPE circuit, 5,16 such as clotting of circuits, high transmembrane pressure, and access issues. Patient complication was defined as any deterioration in the patient's clinical status or laboratory parameters that required intervention $3,5,13,17$ during the mTPE procedure, such as hypocalcaemia that required treatment (ionised calcium $\leq 1.0 \mathrm{mmol} / \mathrm{L}$ ), hypotension (mean arterial pressure $<65 \mathrm{mmHg}$ ), hypoxia (oxygen saturation measured by pulse oximetry $\left.\left[\mathrm{SpO}_{2}\right]<95 \%\right)$, rash and itching. In addition, we compared ICU and non-ICU (ward) patients for any difference in baseline characteristics and complications.

\section{Statistical analysis}

Categorical variables were presented as numbers and percentages, and continuous variables were described as median and interquartile range (IQR) for non-normally distributed data and mean with standard variation (SD) for normally distributed data. SPSS 17.0 was used for statistical analysis. One-way analysis of variance (ANOVA) was used to determine statistically significant differences between continuous variables, and the $\chi^{2}$ test was used to determine statistically significant differences between categorical variables. Logistical regression analysis was used to predict factors contributing to complications during mTPE treatment, such as age, gender, number and duration of $\mathrm{mTPE}$, blood flow rate, methods of anticoagulation, replacement fluid type, ionised calcium level and different diagnostic categories. We used odds ratios (ORs) and 95\% confidence intervals ( $\mathrm{Cls}$ ) with associated $P$ value to report the result of logistic regression. $P<0.05$ was used to assess statistical significance.

\section{Results}

During the study period, 674 mTPE treatments were performed in 140 patients (71 females, 50.7\%). The characteristics of patients and treatments are listed in Table 1. The mean age of patients was 53.1 years (SD, 17.7) and the mean exchange volume was $4421.5 \mathrm{~mL}$ (SD, 1210.4), which was an average of 1.25 times plasma volume. The median mTPE treatment per patient was $3(\mathrm{IQR}, 1-5)$, and the mean duration of mTPE treatment was $170.6 \mathrm{~min}$ (SD, 84.8), with an average of 1.25 circuits utilised per treatment.

Out of 674 mTPE treatments, data for 672 mTPE treatments were available for indications (Online Appendix, figure 1). Among all different disease categories, haematological diseases (30.4\%) were the most common indications for mTPE treatment, followed by neurological disease (29.1\%), liver diseases $(14.1 \%)$, vasculitis $(9.8 \%)$, renal diseases $(8 \%)$, and miscellaneous conditions (8.6\%) (necrobiotic xanthogranuloma, 5.5\%; hypertriglyceridaemia-related severe pancreatitis, 2.2\%; severe sepsis, 0.9\%) (Figure 1). Among all mTPE procedures performed, $52 \%$ were a category I indication as per the ASFA classification (Online Appendix, figure 2). Among all mTPE procedures, albumin was the most common replacement fluid (43.3\%), and citrate was the most common mode of anticoagulation (86.1\%) (Online Appendix, table 5).

Out of 674 mTPE treatments, data for 652 mTPE treatments were available for ICU and non-ICU (ward) patients (Online Appendix, table 1). When comparing ICU with non-ICU patients, they were predominately female $(55.7 \% \vee 43.7 \%)$, more patients received fresh frozen plasma (FFP) as a replacement fluid (39.4\% v $14.1 \%)$, more patients had liver disease as an indication for mTPE (16.4\% $v 10.9 \%)$, more patients were not receiving anticoagulation $(11.8 \% \vee 3.0 \%)$, and more had patient-related complications (15.8\% v 9.9\%). There was no difference between the two groups for circuit-related complications, duration of mTPE treatment or exchange volume (Online Appendix, table 1).

Circuit-related complications occurred in $18.6 \%$ of total mTPE treatment. High transmembrane pressure (8.3\%) was the most common circuit complication, followed by 
Table 1. Patient and treatment characteristics

\begin{tabular}{lc} 
& Values \\
\hline Age (years), mean (SD) & $53.1(17.7)$ \\
Weight (kg), mean (SD) & $78.7(19.2)$ \\
Sex, female & $71 / 140(50.7 \%)$ \\
TPE treatment per patient, mean (IQR) & $3(1-5)$ \\
Exchange volume (mL), mean (SD) & $4421.5(1210.4)$ \\
Exchange volume times plasma volume, mean (SD) & $1.25(0.29)$ \\
Therapy time (min), mean (SD) & $170.6(84.8)$ \\
Starting blood flow (mL/min), mean (SD) & $236.3(54.3)$ \\
Maximum blood flow (mL/min), mean (SD) & $283.1(57.61)$ \\
Filtration fraction (\%), mean (SD) & $25(1.5)$ \\
Number of circuits, mean (SD) & $1.25(0.6)$ \\
Citrate target (mmol/L), mean (SD)* & $3(0.28)$ \\
Pre-treatment iCa (mmol/L), mean (SD) & $1.19(0.14)$ \\
Post-treatment iCa (mmol/L), mean (SD) & $1.04(0.16)$ \\
Post-filter iCa (mmol/L), mean (SD)* & $0.44(0.17)$ \\
\hline
\end{tabular}

$\mathrm{i} C \mathrm{C}=$ ionised calcium; $\mathrm{IQR}=$ interquartile range; $\mathrm{SD}=$ standard deviation; $\mathrm{mTPE}=$ membrane-based therapeutic plasma exchange. * For citrate anticoagulation. clotting of circuits (6.4\%), and access catheter dysfunction (3.6\%) (Table 2). On univariate analysis, the predictors of circuit complications were male sex, combined albumin and FFP as a replacement fluid, combined citrate and prostaglandin as anticoagulation and renal disease as an indication for mTPE (Online Appendix, table 3). On multivariate analysis $\left(R^{2}=0.38\right)$, pre-treatment ionised calcium level (OR, 42.2; $95 \% \mathrm{Cl}, \quad 1.8-975.0 ; \quad P=0.02)$, male sex $(\mathrm{OR}, 2.04 ; 95 \% \mathrm{Cl}, 1.04-$ 4; $P=0.04)$, duration of $\mathrm{mTPE}$ treatment $(\mathrm{OR}, 1.02 ; 95 \% \mathrm{Cl}, 1.01-$ $1.02 ; P<0.001)$ and diagnostic categories $(P=0.03$ ) were predictors of circuit complications (Table 3 ).

During mTPE treatment, $87.2 \%$ of patients did not experience any complications. The most common

Figure 1. Indications of membrane-based therapeutic plasma exchange (mTPE) per number of patients $(n=672)$

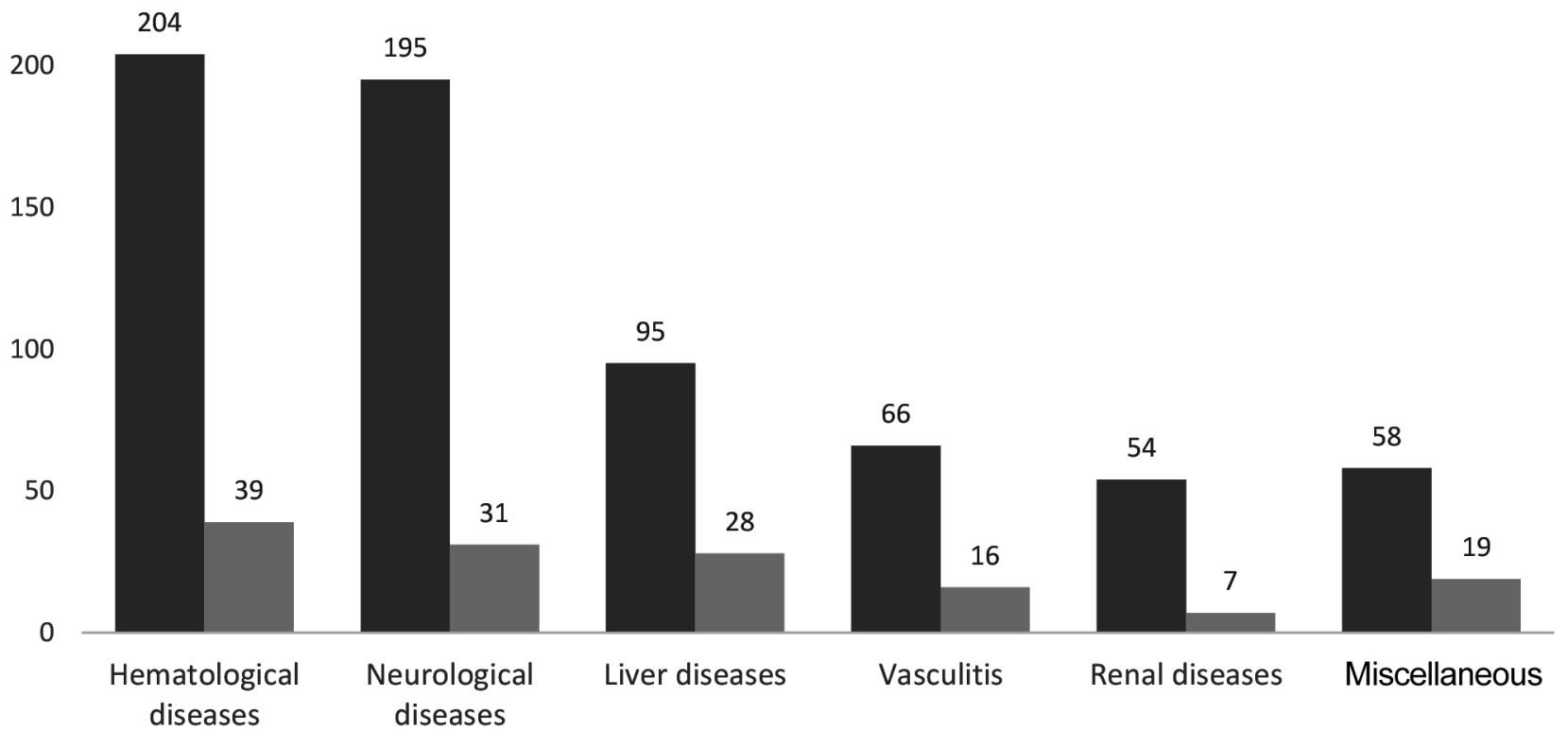

amTPE a Patients 


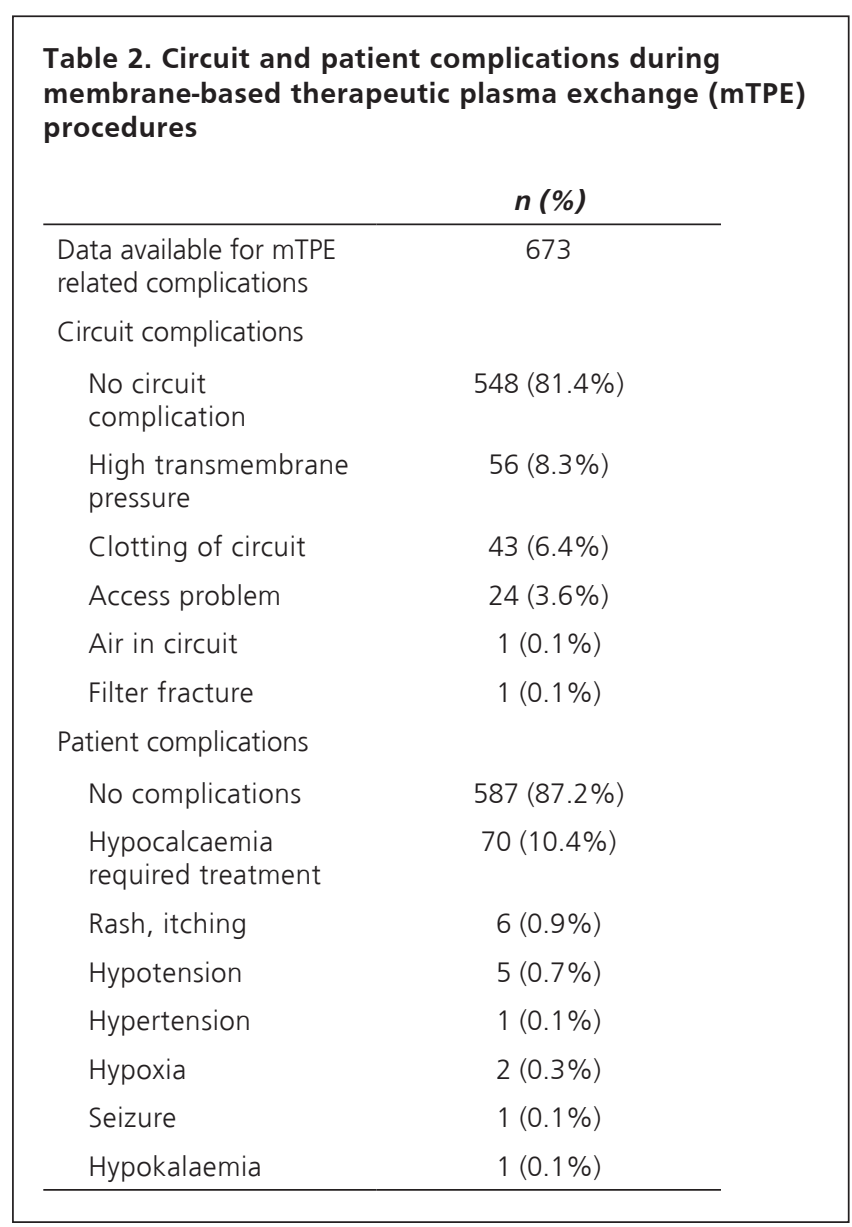

patient complication was hypocalcaemia and required treatment (10.4\%) (Table 2). On univariate analysis, predictors of patient complications were female sex, Combined citrate and prostaglandins as an anticoagulation, FFP as replacement fluid, exchange volume more than one and half times plasma volume and liver disease (Online Appendix, table 4), while higher exchange volume (OR, 8.9; $95 \% \mathrm{Cl}, 1.6-48.7 ; P=0.01)$, replacement fluid type $(P=0.03)$ and lower initial blood flow $(\mathrm{OR}, 0.9 ; 95 \% \mathrm{Cl}$, 0.9-1.0; $P=0.04$ ) were predictors of patient complications on multivariate analysis $\left(R^{2}=0.31\right)$ (Table 4).

\section{Discussion}

With 674 mTPE treatments performed on 140 patients, we present the largest dataset on the use of mTPE at an Australian tertiary care ICU. Neurological and haematological diseases accounted for more than half of our mTPE treatments. Higher pre-treatment ionised calcium level, male sex, duration of mTPE and diagnostic categories were predictors of circuit complications, while higher exchange volume, replacement fluid type, and lower initial blood flow were predictors of patient complications during MTPE.

We used MTPE for 31 different indications, with neurological and haematological diseases being indications for $57 \%$ of mTPE treatment. Our indications of MTPE were similar to the previously reported literature for the use of TPE . 10,12,15,17,18 Our hospital is the major referral centre for liver transplant, which explains a higher rate of liver disease $(15.3 \%$ of total mTPE treatment) as an indication for MTPE. We reviewed our practice of MTPE with the recently published guideline by ASFA. ${ }^{12}$ More than $50 \%$ of our mTPE indications were category I indications, which are diseases for which mTPE is accepted as first line therapy.

There have been a few studies that have reported patientrelated complications during ITPE, recorded between $4 \%$ and $36 \%, 8,11-13,17-22$ Most of these adverse events during MTPE are mild to moderate, and severe complications are rare. ${ }^{18}$ We found that $12.8 \%$ of patients developed complications during MTPE. In our practice, severe complications were only $1.1 \%$ of total mTPE treatment (hypotension [mean arterial pressure $<65 \mathrm{mmHg}$ ], $0.7 \%$; hypoxia, $0.3 \%$; seizure, $0.1 \%$ ) (Table 2 ), which was similar to previously reported studies. $13,17,18,21,22$ On multivariate analysis, we found that higher exchange volume, replacement fluid type, and lower initial blood flow were associated with patient complications. Exchange volume was calculated based on actual body weight and haematocrit. ${ }^{23}$ The average exchange volume in our study was $4.4 \mathrm{~L}$, which was higher than reported in the previous trials. ${ }^{11,17,22}$ FFP was used as a replacement fluid in $57.6 \%$ of patients treated, which may explain the contribution of replacement fluid as a cause of patient complications. Although the effect of blood flow on patient complications was statistically significant $(P=0.04)$, the actual contribution of blood flow on patient complications would be clinically non-significant. The most common complication was hypocalcaemia $(10.4 \%)$, likely due to the use of citrate as regional anticoagulation for $\mathrm{MTPE}(86.1 \%)$, use of FFP as a replacement fluid (FFP also contains citrate), and higher exchange volume.

There are only a few studies that have reported circuit complications during TPE. ${ }^{13,17}$ In this study, circuit-related complications occurred in $18.6 \%$ of mTPE treatments. No previous studies have reported risk factors contributing to the circuit complications during mTPE. Circuit complications can lead to blood loss, may increase the duration of the MTPE treatment and may eventually increase the cost of mTPE treatment. Underlying procoagulant state, increased viscosity, disease-induced haemolysis, and use of FFP as replacement fluid are possible causative factors that can contribute to circuit complications due to different disease 


\begin{tabular}{|c|c|c|c|}
\hline & Values & Odds ratio $(95 \% \mathrm{Cl})$ & $P$ \\
\hline Age (years), mean (SD) & $53.1(17.7)$ & $0.99(0.97-1.00)$ & 0.243 \\
\hline Weight (Kg), mean (SD) & $78.7(19.2)$ & $1.01(0.99-1.03)$ & 0.236 \\
\hline Sex, female & $71 / 140(50.7 \%)$ & $2.04(1.04-4.0)$ & 0.038 \\
\hline mTPE number, median (IQR) & $3(1-5)$ & $1.01(0.97-1.05)$ & 0.520 \\
\hline Duration of mTPE (min), mean (SD) & $170.6(84.8)$ & $1.02(1.01-1.02)$ & $<0.001$ \\
\hline Initial blood flow (mL/min), mean (SD) & $236.3(54.3)$ & $1.00(0.99-1.01)$ & 0.418 \\
\hline Anticoagulation & & & 0.376 \\
\hline Citrate & $580 / 673(86.1 \%)$ & 1 (reference) & \\
\hline Heparin & $18 / 673(2.7 \%)$ & $0.0(0.0-0.0)$ & 0.99 \\
\hline Citrate + Heparin & $9 / 673(1.3 \%)$ & $2.60(0.40-16.89)$ & 0.317 \\
\hline Citrate + PGE1 & $4 / 673(0.6 \%)$ & $0.05(0.00-1.28)$ & 0.069 \\
\hline No anticoagulation & $62 / 673(9.2 \%)$ & $0.0(0.0-0.0)$ & 0.99 \\
\hline Pre-treatment iCa (mmol/L), mean (SD) & $1.20(0.14)$ & $42.24(1.83-975.01)$ & 0.019 \\
\hline Post-treatment iCa (mmol/L), mean (SD) & $1.04(0.16)$ & $0.90(0.106-7.70)$ & 0.926 \\
\hline Post-filter iCa (mmol/L), mean (SD) & $0.44(0.17)$ & $0.92(0.074-11.35)$ & 0.945 \\
\hline Exchange volume times plasma volume, mean (SD) & $1.2(0.29)$ & $0.312(0.07-1.33)$ & 0.115 \\
\hline Replacement fluid & & & 0.054 \\
\hline Albumin & $285 / 673(42.3 \%)$ & 1 (reference) & \\
\hline FFP & 196/673 (29.1\%) & $0.34(0.12-0.99)$ & 0.047 \\
\hline Albumin + FFP & 192/673(28.5\%) & $1.21(0.62-2.39)$ & 0.579 \\
\hline Diagnostic categories & & & 0.032 \\
\hline Neurological disease & $31 / 140(22.1 \%)$ & 1 (reference) & \\
\hline Haematological disease & $39 / 140(27.9 \%)$ & $4.00(0.42-37.93)$ & 0.227 \\
\hline Liver disease & $28 / 140(20 \%)$ & $3.035(0.32-28.51)$ & 0.331 \\
\hline Vasculitis & $16 / 140(11.4 \%)$ & $3.00(0.30-30.21)$ & 0.352 \\
\hline Hypertriglyceridemia/pancreatitis & $13 / 140(9.3 \%)$ & $4.19(0.38-46.0)$ & 0.241 \\
\hline Renal disease & $7 / 140(5 \%)$ & $14.51(1.44-146.04)$ & 0.023 \\
\hline Miscellaneous & $6 / 140(4.3 \%)$ & $68.64(1.67-2819.52)$ & 0.026 \\
\hline
\end{tabular}

FFP = fresh frozen plasma; iCa = ionised calcium; IQR = interquartile range; $m$ TPE = membrane-based therapeutic plasma exchange, $P G E 1=$ prostaglandin E1 (alprostadil); SD = standard deviation.

categories. A higher pre-treatment ionised calcium level requires more citrate dosing to compensate for higher calcium, which may account for more rapid filter clotting. Higher exchange volume can be a cause of the longer duration of MTPE treatment (the average duration of mTPE treatment was $170.6 \mathrm{~min}$ ), which can lead to more circuit-related complications. We found that males were associated with higher odds of circuit complications than females, which might be due to longer duration of mTPE treatment compared with females (178 min $v 162$ min for males $v$ females respectively; $P=0.011$ ) (Online Appendix, table 5), and higher body weight than females $(84.9 \mathrm{~kg} \vee 73.2 \mathrm{~kg}$ for males $v$ females respectively; $P=0.0001$ ) (Online Appendix, table 2). This weight difference may lead to more access catheter dysfunction in males. Moreover, there were increased use of FFP and more MTPE treatments without the use of anticoagulation in females. This may indicate increased coagulopathy in females and may explain the fewer circuit complications in females compared with males (Online Appendix, table 5). However, the exact mechanism is uncertain. 
Table 4. Patient complications: multivariate analysis

\begin{tabular}{|c|c|c|c|}
\hline & Values & Odds ratio $(95 \% \mathrm{Cl})$ & $P$ \\
\hline Age (years), mean (SD) & $53.1(17.7)$ & $0.99(0.98-1.02)$ & 0.727 \\
\hline Weight (kg), mean (SD) & $78.7(19.2)$ & $0.99(0.96-1.01)$ & 0.385 \\
\hline Sex, female, mean (SD) & $71 / 140(50.7 \%)$ & $0.94(0.87-1.00)$ & 0.210 \\
\hline mTPE number, median (IQR) & $3(1-5)$ & $0.94(0.87-1.00)$ & 0.082 \\
\hline Duration of mTPE (min), mean (SD) & $170.6(84.8)$ & $1.00(0.99-1.00)$ & 0.639 \\
\hline Initial blood flow (mL/min), mean (SD) & $236.3(54.3)$ & $0.99(0.98-1.00)$ & 0.044 \\
\hline Anticoagulation & & & 0.742 \\
\hline Citrate & $580 / 673(86.1 \%)$ & 1 (reference) & \\
\hline Heparin & $18 / 673(2.7 \%)$ & $4.32(0.25-73.93)$ & 0.313 \\
\hline Citrate + heparin & $9 / 673(1.3 \%)$ & $0.00(0.0-0.0)$ & 0.99 \\
\hline Citrate + PGE1 & $4 / 673(0.6 \%)$ & $4.15(0.17-99.89)$ & 0.381 \\
\hline No anticoagulation & $62 / 673(9.2 \%)$ & $0.0(0.0-0.0)$ & 0.99 \\
\hline Pre-treatment iCa (mmol/L), mean (SD) & $1.20(0.14)$ & $0.47(0.03-7.91)$ & 0.598 \\
\hline Post-treatment iCa (mmol/L), mean (SD) & $1.04(0.16)$ & $0.12(0.01-1.23)$ & 0.074 \\
\hline Post-filter iCa (mmol/L), mean (SD) & $0.44(0.17)$ & $0.17(0.01-2.12)$ & 0.168 \\
\hline Exchange volume times plasma volume, mean (SD) & $1.2(0.29)$ & $8.95(1.64-48.72)$ & 0.011 \\
\hline Replacement fluid & & & 0.026 \\
\hline Albumin & $285 / 673(42.3 \%)$ & 1 (reference) & \\
\hline FFP & 196/673 (29.1\%) & $4.13(1.44-11.86)$ & 0.008 \\
\hline Albumin + FFP & $192 / 673(28.5 \%)$ & $1.53(0.61-3.85)$ & 0.366 \\
\hline Diagnostic categories & & & 0.610 \\
\hline Neurological disease & $31 / 140(22.1 \%)$ & 1 (reference) & \\
\hline Haematological disease & $39 / 140(27.9 \%)$ & $1.74(0.57-5.35)$ & 0.332 \\
\hline Liver disease & $28 / 140(20 \%)$ & $4.75(1.16-19.52)$ & 0.031 \\
\hline Vasculitis & $16 / 140(11.4 \%)$ & $2.20(0.51-9.67)$ & 0.294 \\
\hline Hypertriglyceridemia/pancreatitis & $13 / 140(9.3 \%)$ & $1.61(0.32-8.06)$ & 0.559 \\
\hline Renal disease & $7 / 140(5 \%)$ & $0.00(0.0-0.0)$ & 0.99 \\
\hline Miscellaneous & $6 / 140(4.3 \%)$ & $2.06(0.31-13.66)$ & 0.455 \\
\hline
\end{tabular}

FFP = fresh frozen plasma; $i \mathrm{Ca}=$ ionised calcium; $\mathrm{QQR}=$ interquartile range; $\mathrm{mTPE}=$ membrane-based therapeutic plasma exchange; $\mathrm{PGE} 1=$ prostaglandin E1 (alprostadil); SD = standard deviation.

\section{Strength}

To our knowledge, this is the largest published data on the use of mTPE in the ICU from Australia. We compared our practice with the recent ASFA guidelines and predicted risk factors for complications during mTPE. Our data can be used as a benchmark for comparison of clinical practice and complications of mTPE in the ICU. This large dataset can help design both local and national guidelines, which can help standardise clinical practice, predict modifiable risk factors, and identify high risk groups for MTPE, therefore increasing the safety and efficacy of the MTPE treatment.

\section{Limitations}

Although it is a single-centre study, our large database represents the use of mTPE for 6 years with a wide variety of different indications, suggesting it can be generalised to the use of MTPE in the ICU. We did not analyse the difference between prescribed and actual plasma volume, pre-procedural haematocrit, post-treatment coagulation profile, and cost analysis.

We believe our study can provide a platform for a future national registry on MTPE use in the ICU, which may increase the safety and efficacy of the procedure. 


\section{ORIGINAL ARTICLES}

\section{Conclusion}

In our large single-centre observational study, we report that MTPE is a safe procedure in the ICU and can be done efficiently by an ICU team. Haematological and neurological diseases accounted for $59 \%$ of our mTPE treatments. Male sex, higher pre-treatment ionised calcium level, duration of MTPE and diagnostic categories were predictors of circuit complications while replacement fluid type, lower initial blood flow and higher exchange volume were predictors of patient complications during mTPE.

\section{Competing interests}

All authors declare that they do not have any potential conflict of interest in relation to this manuscript.

\section{Author details}

Mitul P Chavda ${ }^{1}$

Alpesh Patel ${ }^{1}$

Shailesh Bihari ${ }^{1,2}$

1 Intensive Care Unit, Flinders Medical Centre, Adelaide, SA, Australia.

2 College of Medicine and Public Health, Flinders University, Adelaide, SA, Australia.

Correspondence: drmitulchavda@yahoo.com

doi: https://doi.org/10.51893/2022.1.OA6

\section{Reference}

1 Yilmaz AA, Can OS, Oral $M$, et al. Therapeutic plasma exchange in an intensive care unit (ICU): a 10-year, singlecenter experience. Transfus Apher Sci 2011; 45: 161-6.

2 Paton E, Baldwin IC. Plasma exchange in the intensive care unit: a 10-year retrospective audit. Aust Crit Care 2014; 27: 139-44.

3 Lemaire A, Parquet N, Galicier L, et al. Plasma exchange in the intensive care unit: Technical aspects and complications. J Clin Apher 2017; 32: 405-12.

4 Calça R, Gaspar A, Santos A, et al. Therapeutic plasma exchange in patients in a Portuguese ICU. Port J Nephrol Hypert 2020; 34: 67-71.

5 Szczeklik W, Wawrzycka K, Włudarczyk A, et al. Complications in patients treated with plasmapheresis in the intensive care unit. Anaesthesiol Intensive Ther 2013; 45: 7-13.

6 Ranganathan L, Menon R, Ramakrishnan N, et al. Therapeutic Plasma Exchange Practices in Intensive Care Unit. Indian J Crit Care Med 2019; 23: 336-8.
7 Stahl K, Hadem J, Schneider A, et al. Therapeutic plasma exchange in acute liver failure. J Clin Apher 2019; 34: 589-97.

8 Stahl K, Schmidt JJ, Seeliger B, et al. Effect of therapeutic plasma exchange on endothelial activation and coagulationrelated parameters in septic shock. Crit Care 2020; 24: 71.

9 Keith P, Wells A, Hodges J, et al. The therapeutic efficacy of adjunct therapeutic plasma exchange for septic shock with multiple organ failure: a single-center experience. Crit Care 2020; 24: 518.

10 Samtleben W, Mistry-Burchardi N, Hartmann B et al. Therapeutic plasma exchange in the intensive care setting. Ther Apher 2001; 5: 351-7.

11 Fernandez J, Gratacos-Ginès J, Olivas $P$ et al. Plasma exchange: an effective rescue therapy in critically ill patients with coronavirus disease 2019 infection. Crit Care Med 2020; 48: 1350-5.

12 Padmanabhan A, Connelly-Smith L, Aqui N, et al. Guideline on the use of therapeutic apheresis in clinical practice evidence-based approach from the writing committee of the American Society for Apheresis: the eighth special issue. J Clin Apher 2019; 34: 171-354.

13 Mörtzell Henriksson M, Newman E, Witt V, et al. Adverse events in apheresis: an update of the WAA registry data. Transfus Apher Sci 2016; 54: 2-15.

14 Redant S, De Bels D, Ismaili K, Honoré PM. Membrane-based therapeutic plasma exchange in intensive care. Blood Purif 2020; 50: 290-7.

15 Daga Ruiz D, Fonseca San Miguel F, González de Molina FJ, et al. Plasmapheresis and other extracorporeal filtration techniques in critical patients. Med Intensiva 2017; 41: 174-87.

16 Gashti CN, Andreoli DC, Patel D. Membrane-based therapeutic plasma exchange (mTPE): technical and clinical experience. Clin Apher 2018; 33: 38-45.

17 Shemin D, Briggs D, Greenan M. Complications of therapeutic plasma exchange: a prospective study of 1727 procedures. J Clin Apher 2007; 22: 270-6.

18 Basic-Jukic N, Kes P, Glavas-Boras S, et al. Complications of therapeutic plasma exchange: experience with 4857 treatments. Ther Apher Dial 2005; 9: 391-5.

19 Reeves Winters J. The mechanism of action of plasma exchange. Br J Haematol 2013; 164: 342-51.

20 Bramlage CP, Schröder K, Bramlage P, et al. Predictors of complications in therapeutic plasma exchange. J Clin Apher 2009; 24: 225-31.

21 Stegmayr B, Ptak J, Wikström B. World apheresis registry report. Transfus Apher Sci 2007; 36: 13-6.

$22 \mathrm{Wu}$ MJ, Shu KH, Cheng CH, Lian JD. Complications of membrane-filtration plasma exchange. Zhonghua Yi Xue Za Zhi (Taipei) 1997; 60: 147-54.

23 Kaplan AA. A simple and accurate method for prescribing plasma exchange. ASAIO Trans 1990; 36: 597-9. 